\title{
CAPTURA, TRANSPORTE Y ACLIMATACIÓN DE JUVENILES Y ADULTOS DE RÓBALO Centropomus undecimalis (Bloch, 1792)
}

\author{
CAPTURE, TRANSPORT AND ACCLIMATION OF JUVENILES AND ADULTS OF \\ COMMON SNOOK Centropomus undecimalis (Bloch, 1792)
}

Carmen Polonía-Rivera D, Saeko Gaitán ${ }^{\text {CuLAC }}$, Nicolás Chaparro-Muñoz ${ }^{\text {CuLAC }}$ y Natalia Villamizar D

\begin{abstract}
RESUMEN
El presente estudio se realizó en el marco de una investigación sobre la viabilidad del cultivo del róbalo Centropomus undecimalis, como una solución socioeconómica para las poblaciones costeras, que a su vez ayudaría a disminuir la presión de la pesca sobre este recurso. Respondiendo al objetivo de estandarizar un protocolo específico de captura, transporte y aclimatación de juveniles y adultos, se describen los procesos para lograr individuos completamente aclimatados a condiciones de cautiverio, ya sea en estanques en tierra con agua dulce o en tanques con circulación de agua de mar, a partir de peces provenientes de la Ciénaga Grande de Santa Marta (Colombia). La captura de juveniles se llevó a cabo en las áreas adyacentes a bosques de manglar, con atarraya de $8 \mathrm{~mm}$ (tamaño de malla) y en canoas dotadas de tanques con agua del medio; la captura de adultos se realizó con trampas o jaulones fijados al fondo. El método más efectivo para el transporte de juveniles y adultos de róbalo (100 \% de supervivencia) fue la utilización de bolsas plásticas provistas de 301 de agua del lugar de captura, oxígeno inyectado y hielo. La densidad óptima fue de $30 \mathrm{~g} / \mathrm{L}$ y los peces se anestesiaron con eugenol al 85 \%, aplicando 0,1 ml por cada $10 \mathrm{l}$ de agua para disminuir el estrés. La aclimatación se llevó a cabo disminuyendo o aumentando la salinidad en 5 cada 3 horas y aplicando un tratamiento profiláctico de formalina previo a su ingreso a los sistemas receptores.
\end{abstract}

PALABRAS CLAVE: acuicultura; adaptación; salinidad; estanques; tanques

\begin{abstract}
In the framework of a study on common snook Centropomus undecimalis culture viability as a socioeconomic solution for the coastal communities that in turn will decrease the fishing pressure on wild snook stocks, this study was aimed to standardize a specific protocol for capture, transport and acclimation of juvenile and adult snook. Therefore, this paper describes the process to obtain fish fully acclimatized to captivity conditions, either in rustic earthen ponds with fresh water or in tanks with circulating seawater, from fish captured at the Ciénaga Grande de Santa Marta, Colombia. Juveniles were captured near mangrove forests by using cast nets with $8 \mathrm{~mm}$ mesh size and boats provided with tanks filled with local water. Snook adults were captured with traps or cages fixed at the bottom. The most effective method for juvenile and adult fish transportation with a $100 \%$ survival rate was using $30 \mathrm{l}$ plastic bags filled with local water, injecting oxygen and adding ice. Optimal density was of $30 \mathrm{~g} / \mathrm{L}$ and fish were lightly anesthetized to reduce stress. Acclimation was obtained by decreasing or increasing salinity by 5 every three hours and applying prophylactic formalin baths before moving fish to the new systems (freshwater ponds or sea water tanks).
\end{abstract}

KEY WORDS: aquaculture; adaptation; salinity; ponds; tanks

Dirección de los autores:

Programa de Ingeniería Pesquera - Universidad del Magdalena, Carrera 32 \# 22-08, Santa Marta, Colombia; e-mail: ccpr1991@gmail.com, ORCID: 0000-0002-3032-428X (C.P.R.). Grupo de Investigación Biodiversidad y Ecología Aplicada, Universidad del Magdalena, Carrera 32 \# 22-08, (5) 421 7940 ext. 2295. Santa Marta, Colombia (S.G.) (N.C.M.) ORCID: 0000-0002-3032-428X (N.V.). 
El róbalo Centropomus undecimalis se distribuye ampliamente a lo largo de la costa oeste del océano Atlántico y el mar Caribe (Nelson, 2006). Se caracteriza por ser gregario, carnívoro, catádromo y eurihalino (Tringali y Bert, 1996; Tringali et al., 1999; Taylor et al., 2000; Chaparro et al., 2008; Perera-García et al., 2011). El róbalo es hermafrodita protándrico, habita aguas estuarinas y migra al mar para reproducirse bajo salinidades superiores a 30 (Grier, 1985; Tucker, 1987; Taylor et al., 1998; Perera-García et al., 2008; 2011; 2013).

Debido a factores como el deterioro ambiental de su hábitat, la sobreexplotación y una inexistente acuicultura, en Colombia se le ha declarado como una especie en categoría de Vulnerable -VU- (Acero et al., 2002). Por tanto, se hacen necesarios estudios que evalúen la viabilidad de su cultivo y en caso positivo, que conlleven al establecimiento de un paquete tecnológico que pueda ser ofrecido a las comunidades locales como alternativa socioeconómica sostenible, que además pueda servir para disminuir la presión que ejerce la pesca sobre la especie. Por lo anterior, este estudio busca estandarizar un protocolo para los procesos de captura, transporte y aclimatación del róbalo en sus etapas juveniles y adultas, tanto en agua dulce como marina, ya sea en estanques o en tanques.

Las capturas se realizaron en la Ciénaga Grande de Santa Marta (CGSM) y los sistemas de cultivo se ubicaron en dos dependencias de la Universidad del Magdalena (Santa Marta): en la Granja Acuícola Experimental (sede principal), donde se asignó un estanque de agua dulce de $600 \mathrm{~m}^{2}$ para tal fin, y en el Centro de Desarrollo Pesquero (sede Taganga), específicamente en un tanque de agua de mar de $8 \mathrm{~m}^{3}$ ubicado en el laboratorio de peces. El siguiente protocolo se ha establecido después de haber aplicado varios métodos para la aclimatación y transporte, en donde se obtuvieron diversos porcentajes de supervivencia. A continuación se describe la metodología adoptada para obtener un $100 \%$ de supervivencia.

La primera fase del proceso fue la captura de los juveniles y adultos de róbalo, la cual fue realizada por pescadores de la CGSM, en la zona de la Boca de la Barra, en los sitios denominados como Punta de Cerro y Los Morritos, caracterizado por ecosistemas de bosques de manglar (Figura 1). Las faenas iniciaron a las seis de la mañana, en canoas sin motor provistas de un tanque con agua del lugar para introducir los peces capturados. 62 El arte de pesca utilizado para la captura de los juveniles de robalo fue la atarraya (tamaño de malla de $8 \mathrm{~mm}$ ), mientras que la captura de los adultos involucró el anclaje de una trampa o jaulón de $1 \mathrm{~m}^{3}$, construido con malla de 25,4 mm. En el interior y exterior de las jaulas se colocaron ramas y raíces de manglar con el propósito de atraer peces pequeños y crustáceos, los cuales hacen parte de la dieta del róbalo. La captura de adultos de la especie involucró mantener la trampa sumergida por un periodo de tres a cuatro semanas.

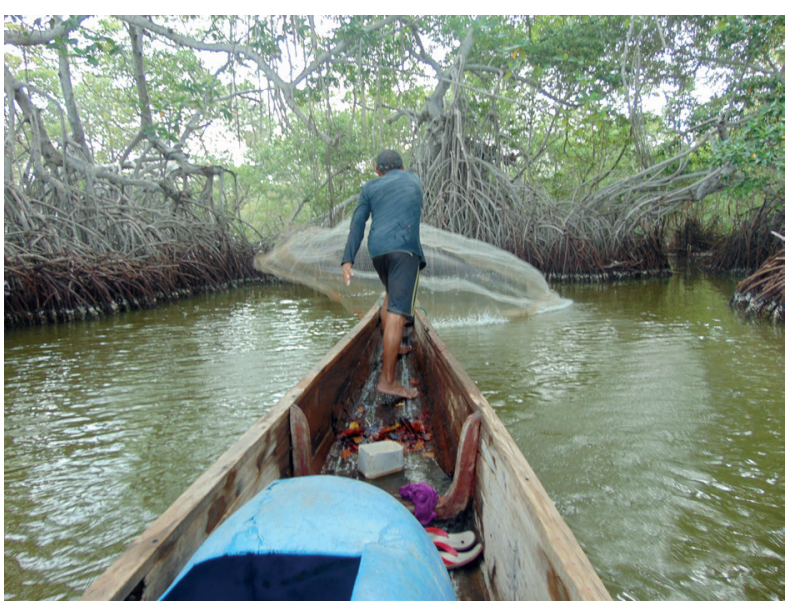

Figura 1. Lance de pesca con atarraya cerca de los manglares para capturar juveniles de róbalos. Salida de campo a la CGSM, sector Punta de Cerro.

La segunda fase fue la estandarización del proceso de aclimatación, la cual se realizó en un tanque de $1 \mathrm{~m}^{3}$ con agua de la CGSM a una salinidad promedio de $33,9 \pm 3,3$. En el caso de róbalos destinados al cultivo en estanques de agua dulce, se realizó un descenso de la salinidad agregando agua dulce hasta alcanzar una salinidad de 5 cada tres horas, logrando valores de salinidad finales de 0 - 0,5. El mismo procedimiento se realizó con el agua de mar para incrementar la salinidad a 37 cuando los peces fueron trasladados a tanques con agua de mar. Durante el proceso de aclimatación se suministró aireación constante y cada hora se monitorearon los parámetros oxígeno disuelto, temperatura, $\mathrm{pH}$ y salinidad.

La tercera etapa consistió en el transporte desde la CGSM hasta las dependencias de la Universidad del Magdalena. Una vez finalizado el proceso de aclimatación a la salinidad, se disminuyó el volumen hasta 50 L, para permitir el desplazamiento de los peces. Se agregó anestésico eugenol al $85 \%$, en una dosis de $0,1 \mathrm{ml}$ por cada $10 \mathrm{l}$ de agua. En 5 minutos aproximadamente los 
peces estuvieron sedados (sin movimientos natatorios y posición ventral). Posteriormente, los peces se recolectaron utilizando paños húmedos para evitar la pérdida de mucus y / o escamas y se introdujeron en bolsas de polietileno con un capacidad de $30 \mathrm{l}$ de agua (densidad de $30 \mathrm{~g}$ / L), a las que también se les agregó eugenol (dosis igual). Cada bolsa fue inyectada con oxígeno a razón de $24 \mathrm{mg} / \mathrm{L}$. La bolsa con los peces se dispuso, a su vez, dentro de una bolsa negra con las mismas características, con el fin de impedir la entrada de luz, provista también de fundas de gel refrigerante para contrarrestar la subida de temperatura durante el transporte. Finalmente, las bolsas fueron introducidas en recipientes plásticos para asegurar su estabilidad dentro del vehículo. En el presente estudio se realizaron transportes que tardaron entre media y una hora.

Una vez en las instalaciones de la Universidad, las bolsas fueron introducidas a cada sistema (estanque 0 tanque), para una aclimatación final a la temperatura (Figuras 2a y 2b). Finalizada la aclimatación, los peces fueron pesados, medidos y sometidos a un tratamiento profiláctico constituido por un baño de formalina (150 ppm) por 15 minutos (Benetti, 1997).

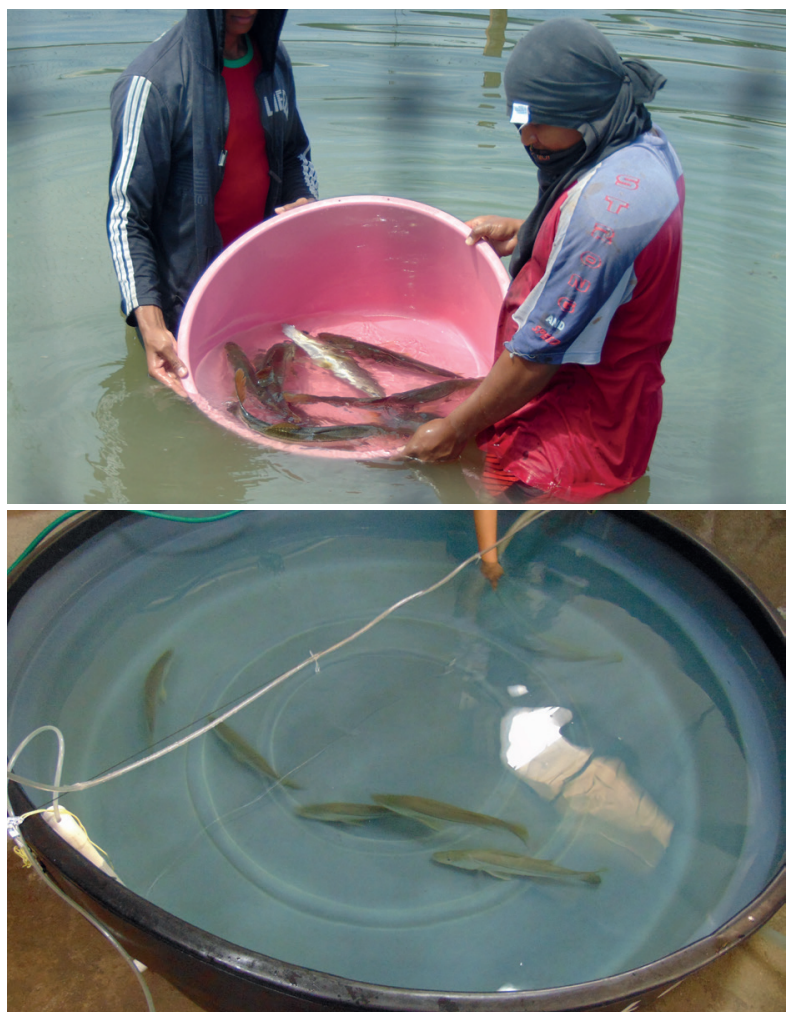

Figura 2. Aclimatación final de Centropomus undecimalis a la temperatura de los sistemas de cultivo receptores: estanque (a) y tanques (b).
Posteriormente se realizó un seguimiento durante un mes, en el cual los róbalos fueron alimentados ad libitum con pescado fresco (Opisthonema oglinum). La supervivencia al final de este periodo de seguimiento fue del $100 \%$, tanto para juveniles como para adultos.

\section{AGRADECIMIENTOS}

Este trabajo se desarrolló en el marco del proyecto titulado: "Contribuir al fortalecimiento de la maricultura del Departamento mediante la implementación de un protocolo de producción de semilla de pepino de mar y fortalecer el paquete tecnológico de pectínidos y los aspectos fisiológicos preliminares y morfológicos relacionados con la reproducción del róbalo (Centropomus undecimalis) y su cultivo experimental en estanques de agua dulce", Convenio Especial de Cooperación No. 090 del 05 de noviembre de 2013, suscrito entre la Universidad del Magdalena y la Gobernación del Departamento del Magdalena.

\section{BIBLIOGRAFÍA}

Acero, A., Mejía, L. y Santos-Acevedo, M. 2002. Centropomus undecimalis. En: Mejía, L. y Acero, A., Editores. Libro rojo de peces marinos de Colombia. INVEMAR. Instituto de Ciencias Naturales-Universidad Nacional de Colombia, Ministerio del Medio Ambiente. La serie de libros rojos de especies amenazadas de Colombia. Bogotá, Colombia.

Benetti, D. 1997. Spawning and larval husbandry of flounder Paralichthys woolmani and pacific yellowtail Seriola mazatlana, new candidate species for aquaculture. Aquaculture Journal 155: 307-318.

Chaparro, N., Madariaga, E., Gaitán, S. y Acero, A. 2008. Captura, transporte, aclimatación y aspectos reproductivos en laboratorio del róbalo Centropomus sp. Convenio Universidad del Magdalena. Memorias del Simposio Biocaribe.

Grier, H. 1985. Reproduction of the common snook, Centropomus undecimalis: Structure of the testis I reproductive males. Florida, USA. In: Proc. World Marine Soc. 16th Annual Meeting 13-17.

Nelson, J. 2006. Fishes of the world. Wiley Book, Nueva York, EE.UU.

Perera-García, M.A., Mendoza-Carranza, M. y PáramoDelgadillo, S. 2008. Dinámica reproductiva y poblacional del robalo, Centropomus undecimalis (Perciformes: Centropomidae), en barra San Pedro, Centla, México. Universidad y Ciencia 24: 49-59. 
Perera-García, M.A., Mendoza-Carranza, M., ContrerasSánchez, W.M., Huerta-Ortiz, M. y Pérez-Sánchez, E. 2011. Reproductive biology of common snook Centropomus undecimalis (Perciformes: Centropomidae) in two tropical habitats. Revista de Biología Tropical 59: 669-681.

Perera-García, M.A., Mendoza-Carranza, M., ContrerasSánchez, W.M., Ferrara, A., Huerta-Ortiz, M. y HernándezGómez, R.E. 2013. Comparative age and growth of common snook Centropomus undecimalis (Pisces: Centropomidae) from coastal and riverine areas in southern Mexico. Revista de Biología Tropical 61: 807-819.

Taylor, R., Grier, H. y Whittington, J. 1998. Spawning rhythms of common snook in Florida. Journal of Fish Biology 53: 502-520.
Taylor, R., Whittington, J., Grier, H. y Crabtree, R. 2000. Age, growth, and protandric sex reversal in common snook Centropomus undecimalis from the east and west coasts of Florida. Journal of Fish Bullentin 98: 612-624.

Tringali, M. y Bert, T. 1996. The genetic stock structure in common snook Centropomus undecimalis. Journal of Fish Aquaculture Science 53: 974-984.

Tringali, M., Bert, T. y Seyoum, S. 1999. Genetic identification of centropomine fishes. Transactions of the American Fisheries Society 1: 446-458.

Tucker, J. 1987. Snook and tarpon snook culture and preliminary evaluation for commercial farming. Program Fisheries Culture 49: 49-57.

Fecha de recepción: 25/02/2016

Fecha de aceptación: 28/11/2016

Fecha de publicación: 07/04/2017

Para citar este artículo: Polonía-Rivera, C., Gaitán, S., Chaparro-Muñoz, N. y Villamizar, N. 2017. Captura, transporte y aclimatación de juveniles y adultos de róbalo Centropomus undecimalis

(Bloch, 1792). Revista Intropica Vol. 12(1): 61 - 64. DOI: http://dx.doi.org/10.21676/23897864.2035 